\title{
Metabolic Profiles Distinguish Non-Dampness-Phlegm and Dampness-Phlegm Patterns among Korean Patients with Acute Cerebral Infarction
}

\author{
Min Ho Cha, ${ }^{1,2}$ A. Daniel Jones, ${ }^{2,3}$ Mi Mi Ko, ${ }^{1}$ Chen Zhang, ${ }^{2}$ and Myeong Soo Lee ${ }^{1}$ \\ ${ }^{1}$ Medical Research Division, Korea Institute of Oriental Medicine, 1672 Yuseongdae-ro, Yuseong-gu, \\ Daejeon 305-811, Republic of Korea \\ ${ }^{2}$ Department of Biochemistry and Molecular Biology, Michigan State University, 603 Wilson Road, East Lansing, MI 48824, USA \\ ${ }^{3}$ Department of Chemistry, Michigan State University, 578 South Shaw Lane, East Lansing, MI 48824, USA
}

Correspondence should be addressed to Min Ho Cha; mhchamin@kiom.re.kr

Received 6 November 2012; Accepted 18 February 2013

Academic Editor: MinKyun Na

Copyright (C) 2013 Min Ho Cha et al. This is an open access article distributed under the Creative Commons Attribution License, which permits unrestricted use, distribution, and reproduction in any medium, provided the original work is properly cited.

Traditional Korean Medicine classifies stroke into four subtype patterns according to symptomatic pattern identification: Qi deficiency (QD), Yin deficiency (YD), Dampness-phlegm (DP), and Fire and Heat (FH). This study investigated the difference in metabolic profiles of plasma comparing subjects displaying non-DP and DP patterns. A total of 141 patients with cerebral infarction enrolled in this study were distributed as non-DP $(N=68)$ and DP $(N=73)$. Anthropometric parameters and symptom/sign index were measured. Metabolic profiling was performed using ultrahigh-performance liquid chromatography-mass spectrometry. The Ratio of subjects with slippery pulse was higher in DP pattern, but fine pulse was lower than that in non-DP pattern. As a result of metabolomics analysis, twenty-one metabolites displayed different levels between non-DP and DP patterns. Two were identified as lysophosphatidylcholines (LPCs), LPC(18:2), and LPC(20:3) having an unsaturated acyl chain and showed lower levels in DP pattern than in non-DP pattern $(P=0.015,0.034$, resp.). However, the saturated LPCs, LPC $(18: 0)$ and LPC $(16: 0)$, exhibited slight but statistically insignificant elevation in DP pattern. Our results demonstrated that plasma LPCs with polyunsaturated fatty acid groups were associated with DP pattern and suggest that variation of plasma lipid profiles may serve as potential biomarker for diagnosis of DP pattern.

\section{Introduction}

Pattern Identification (PI) is a unique diagnosis system of traditional medicine practiced in East Asian countries including China, Korea, and Japan and provides information that guides appropriate treatment of patient disease. Previous reports described how traditional Korean medicine (TKM) categorizes stroke as four patterns according to their symptoms and sign: Qi deficiency (QD), Dampness-phlegm (DP), Yin deficiency (YD), and Fire and Heat (FH) [1]. Among them, the DP pattern is attributed to the prevention of Qiblood circulation in the body, resulting in a pathological product [2]. Most notably, the DP pattern is associated with overweight/obesity and dyslipidemia [3-6]. Kim et al. reported that body mass index (BMI) of subjects with DP pattern was significantly increased compared with subjects exhibiting other patterns [5], and Kang et al. showed that the levels of total cholesterol, LDL-cholesterol and triglycerides in serum of subjects displaying the DP pattern were also higher than subjects with non-DP pattern [6].

However, the diagnosis of PI depends on symptoms/sign exhibited by patients, which may be interpreted incorrectly. Therefore, it is necessary to establish scientific evidence to aid accurate diagnosis based on PI. Previously, several studies showing relation between biological factors and PI were reported [7-13]. In particular, NPY and UCP2 and PON1, related with obesity and dyslipidemia, were significantly associated with DP pattern in Korean stroke patients $[8,9,13]$.

Metabolomics provides a powerful approach for global and quantitative assessment of endogenous small molecule 

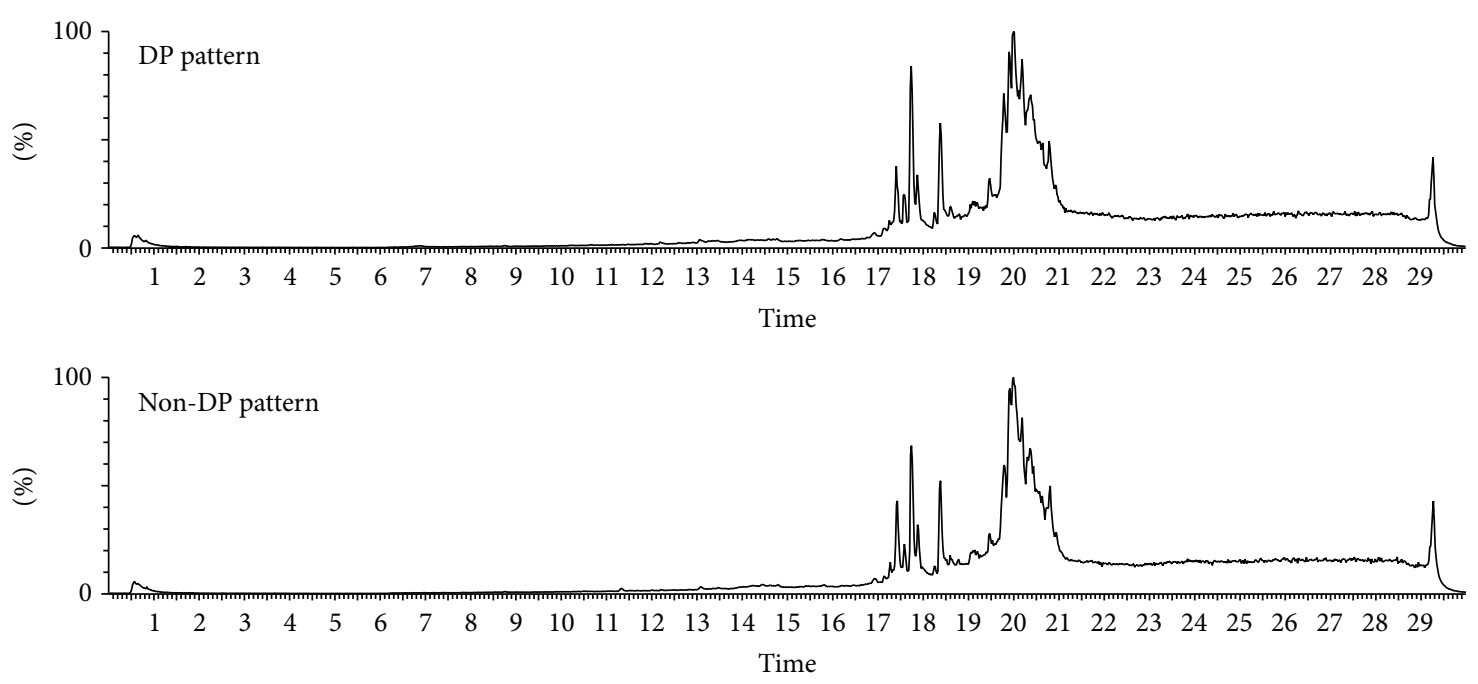

(a)
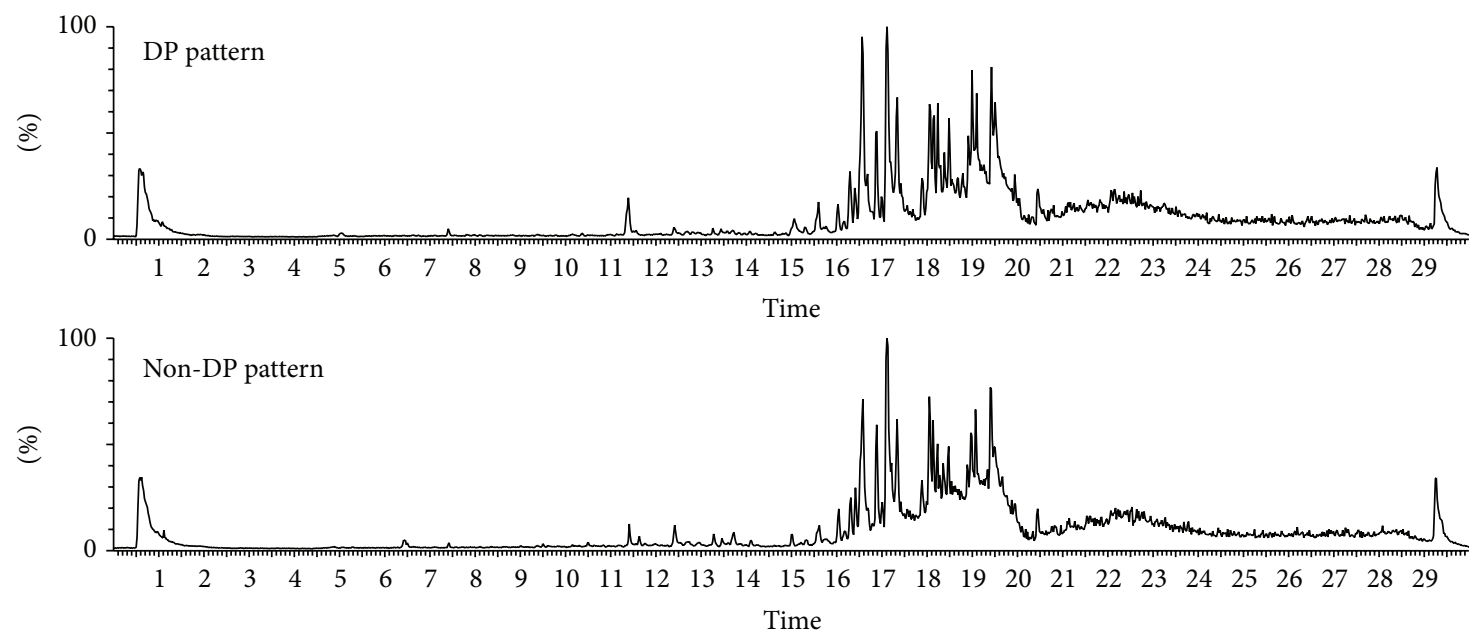

(b)

FIGURE 1: Chromatographic pattern of human plasma metabolite in ESI-positive (a) and ESI-negative mode (b) generated using UHPLC-MS analysis.

metabolites within biological fluids [14] and has been utilized to propose candidate biomarkers of several diseases [15-20]. Metabolites are the small chemical compounds or products involved in various metabolic pathways, and the changes of their levels serve as indicators of changes of pathology or physiology [14]. Recently, metabolomics investigations were performed to explain PI of rheumatoid arthritis and type 2 diabetes in Chinese populations [21, 22].

In this study, we analyzed metabolic profiles in plasma of acute patients with cerebral infarction (CI) by ultrahighperformance liquid chromatography/time-of-flight mass spectrometry (UHPLC-TOFMS) and searched for metabolites differing in levels between DP and non-DP patterns.

\section{Materials and Methods}

2.1. Study Subjects. This study was collected as parts of the project "The Fundamental Study for the Standardization and
Objectification of PI in TKM for Stroke (SOPI-Stroke)" in the Korean Institute of Oriental Medicine (KIOM) [23].

Patients with CI were admitted from 2009 to 2010 to the following Korean oriental medical hospitals participating in this study: Kyung Hee Oriental Medical Center (Seoul), Kyung Hee East-West Neo Medical Center (Seoul), Dong Guk International Hospital (Kyunggi-do), and Dae Jeon Oriental Medical Hospital (Daejeon). Subjects had to be enrolled as CI patients within three days of the onset, and their symptoms were confirmed by imaging diagnosis, using computerized tomography (CT), magnetic resonance imaging (MRI), or magnetic resonance angiography (MRA). CI subtypes were classified as large artery atherosclerosis (LAA), cardioembolism (CE), small vessel occlusion (SVO), stroke of other determined etiology (SOE), and stroke of undetermined etiology (SUE) subtype according to the trial of ORG 10172 in the Acute Stroke Treatment (TOAST) classification. Subjects with subarachnoid, subdural, or epidural hemorrhage were 
TABLE 1: General characteristics of subjects.

\begin{tabular}{|c|c|c|c|}
\hline Characteristics & Non-DP & DP & $P$ \\
\hline$N$ & 68 & 73 & \\
\hline Gender (M/F) & $38(55.9) / 30(44.1)^{*}$ & $36(49.3) / 37(50.7)$ & 0.501 \\
\hline Age (year) & $67.50 \pm 11.43$ & $69.73 \pm 11.07$ & $0.213^{\mathrm{b}}$ \\
\hline TOAST (LAA/CE/SVO/SOE/SUE) & $15(22.1) / 1(1.5) / 43(63.2) / 3(4.4) / 6(8.8)$ & $17(23.3) / 4(5.5) 45(61.6) / 0(0.0) / 7(9.6)$ & 0.300 \\
\hline Weight (kg) & $63.18 \pm 11.49^{\#}$ & $63.67 \pm 10.63$ & $0.799^{\mathrm{a}}$ \\
\hline $\operatorname{BMI}\left(\mathrm{kg} / \mathrm{m}^{2}\right)$ & $24.40 \pm 3.75$ & $24.43 \pm 3.50$ & $0.968^{\mathrm{a}}$ \\
\hline Smoking (none/strop/active) & $33(48.5) / 11(16.2) / 24(35.3)$ & $36(49.3) / 7(9.6) / 30(41.1)$ & 0.470 \\
\hline Drinking (none/stop/active) & $21(30.9) / 11(16.2) / 36(52.9)$ & $25(34.2) / 15(20.5) / 33(45.2)$ & 0.632 \\
\hline \multicolumn{4}{|l|}{ History disease } \\
\hline TIA & $7(10.3)$ & $8(11.1)$ & 1.000 \\
\hline Hypertension & $37(54.4)$ & $36(49.3)$ & 0.614 \\
\hline Hyperlipidemia & $6(8.8)$ & $7(9.6)$ & 1.000 \\
\hline Heart disease & $4(5.9)$ & $2(2.8)$ & 0.429 \\
\hline \multicolumn{4}{|l|}{ Serum parameters } \\
\hline GOT (U/dL) & $23.51 \pm 9.25$ & $22.97 \pm 6.13$ & $0.666^{\mathrm{b}}$ \\
\hline GPT (U/dL) & $20.26 \pm 10.10$ & $20.52 \pm 8.50$ & $0.623^{\mathrm{b}}$ \\
\hline Total cholesterol (mg/dL) & $193.66 \pm 41.89$ & $197.14 \pm 43.83$ & $0.632^{\mathrm{a}}$ \\
\hline Triglyceride (mg/dL) & $192.51 \pm 119.82$ & $187.63 \pm 172.77$ & $0.176^{\mathrm{b}}$ \\
\hline HDL-cholesterol (mg/dL) & $42.97 \pm 10.64$ & $43.34 \pm 10.47$ & $0.735^{\mathrm{b}}$ \\
\hline LDL-cholesterol (mg/dL) & $113.18 \pm 35.32$ & $119.25 \pm 38.19$ & $0.344^{\mathrm{a}}$ \\
\hline Blood sugar (mg/dL) & $116.46 \pm 33.68$ & $117.21 \pm 39.63$ & $0.835^{\mathrm{b}}$ \\
\hline
\end{tabular}

*indicates the number of subjects (\%), and ${ }^{\#}$ indicates the value of mean \pm SD. The $P$ value of categorized variables was calculated by chi-squared test or Fisher's test, and continuous variables were analyzed by a student's $t$-test (a) or Mann-Whitney $U$ test (b) after a normality test and compared with the non-DP group. DP: Dampness-phlegm, BMI: body mass index, TIA: transient ischemic attack, GOT: glutamic oxaloacetic transaminase, GPT: glutamic pyruvate transaminase.

excluded in this study, and subjects with diabetes mellitus and stroke in history and with infectious diseases and liver diseases were also excluded.

After obtaining written informed consent from all subjects, clinical data were collected. This study was approved by the Institutional Review Boards (IRB) of the KIOM and by each of the oriental medical hospitals.

2.2. PI Diagnosis of CI Patients. The symptoms and signs of subjects were collected using "stroke PI case report form" [2], and PI diagnosis of subjects was determined by two expert TKM doctors based on "Korean Standard PIs for StrokeIII" previously reported by Lee et al. [1]. Subjects receiving differing diagnoses from the two doctors were excluded. The number of patients was 68 in non-DP and 73 in DP.

2.3. Plasma Preparation. Preparation of plasma from each subject for UHPLC-MS analysis was performed according to protocols provided by Human Serum Metabolome (HUSERMET) Consortium [24]. Briefly, $200 \mu \mathrm{L}$ of plasma thawed on ice was thoroughly mixed with $600 \mu \mathrm{L}$ methanol and $100 \mu \mathrm{L}$ of internal standard solution and centrifuged at $15000 \mathrm{~g}$ for $15 \mathrm{~min}$ at $25^{\circ} \mathrm{C}$. Supernatant was transferred and completely dried by using centrifugal vacuum evaporator over 4 hours. Dried samples were resolved by adding $100 \mu \mathrm{L}$ of melting solution (acetonitrile/isopropanol/water, $3: 2: 2$ ) and centrifuged at $15000 \mathrm{~g}$ for $15 \mathrm{~min}$ at $25^{\circ} \mathrm{C}$. Supernatant was transferred into vial and used for UHPLC-MS analysis.
2.4. Metabolic Profiling Analysis by UHPLC-MS. A $10 \mu \mathrm{L}$ aliquot of the concentrated plasma extract was injected into an Ascentis Express C18 column $(10 \mathrm{~cm} \times 2.1 \mathrm{~mm}, 2.7 \mu \mathrm{m}$ particles: Supelco, Bellefonte, PA, USA) using a SIL-5000 autosampler (Shimadzu, Tokyo, Japan). The solvent was delivered by LC-20AD pumps (Shimadzu Co., Tokyo, Japan) at a flow rate of $0.4 \mathrm{~mL} / \mathrm{min}$, with solvent $\mathrm{A}$ being $0.15 \%$ aqueous formic acid and solvent $\mathrm{B}$ being acetonitrile/isopropanol $(2: 1)$. Gradient elution was performed and equilibrated with water containing $0.15 \%$ formic acid. Samples were eluted by gradient containing $0.15 \%$ formic acid for $30 \mathrm{~min}$, and metabolites separated by UHPLC were assigned by MS (Waters, Milford, MA, USA). Separate injections were made for analyses conducted ESI-positive and ESI-negative modes.

The voltage of capillary and sampling cone was, respectively, set at $3 \mathrm{kV}$ and $10 \mathrm{~V}$, and the desolvation flow was set to $350 \mathrm{~L} / \mathrm{h}$ at a temperature of $350^{\circ} \mathrm{C}$. The source temperature was set to $100^{\circ} \mathrm{C}$. Collecting range of MS data was $\mathrm{m} / \mathrm{z}$ 100-2000 with a scan time of $0.2 \mathrm{~s}$. The accurate mass and composition for the metabolite ions were calculated by MassLynx 4.1 (Waters, Milford, MA, USA) incorporated in the instrument.

2.5. Data Processing and Identification of Metabolites. Results from UHPLC-MS analyses were processed using automated peak detection, integration, and retention time alignment using MarkerLynx 4.1 software (Waters, Milford, MA, USA), and MS data were assembled into a matrix of integrated 
TABle 2: Difference distribution of symptoms/signs between Dampness-phlegm and non-Dampness-phlegm.

\begin{tabular}{|c|c|c|c|}
\hline Symptoms and signs & Non-DP $(n=68)$ & $\mathrm{DP}(n=73)$ & $P$ \\
\hline Vexation and insomnia & $14(20.59)$ & $9(12.33)$ & 0.1847 \\
\hline Reddened complexion & $27(39.71)$ & $28(38.36)$ & 0.8696 \\
\hline Blood-shot eyes & $8(11.76)$ & $10(13.7)$ & 0.731 \\
\hline Wheezing in the throat with sputum & $14(20.59)$ & $18(24.66)$ & 0.5643 \\
\hline Fetid mouth odor & $15(22.06)$ & $12(16.44)$ & 0.3967 \\
\hline Thirst & $26(38.24)$ & $24(32.88)$ & 0.5063 \\
\hline Red tongue & $28(41.18)$ & $25(34.25)$ & 0.3959 \\
\hline Yellow fur & $13(19.12)$ & $21(28.77)$ & 0.1808 \\
\hline Thick fur & $23(33.82)$ & $36(49.32)$ & 0.0624 \\
\hline Heat vexation in the chest & $14(20.59)$ & $12(16.44)$ & 0.5255 \\
\hline Turbid urine & $8(11.76)$ & $14(19.18)$ & 0.2255 \\
\hline Rapid pulse & $17(25)$ & $19(26.03)$ & 0.8888 \\
\hline Strong pulse & $26(38.24)$ & $33(45.21)$ & 0.4018 \\
\hline Surging pulse & $7(10.29)$ & $4(5.48)$ & 0.2868 \\
\hline Heat vexation and aversion to heat & $20(29.41)$ & $21(28.77)$ & 0.9329 \\
\hline Heat in the palms and soles & $7(10.29)$ & $5(6.85)$ & 0.4639 \\
\hline Vexing heat in the extremities & $4(5.88)$ & $3(4.11)$ & 0.7113 \\
\hline Pale face and red zygomatic site & $13(19.12)$ & $6(8.22)$ & 0.0583 \\
\hline Dry mouth & $40(58.82)$ & $33(45.21)$ & 0.1059 \\
\hline Dry fur & $10(14.71)$ & $19(26.03)$ & 0.0965 \\
\hline Bare and red tongue like a mirror & $4(5.88)$ & $2(2.74)$ & 0.4289 \\
\hline Night sweating & $5(7.35)$ & $9(12.33)$ & 0.3235 \\
\hline Tidal fever & $4(5.88)$ & $2(2.74)$ & 0.4289 \\
\hline Thin & $14(20.59)$ & $17(23.29)$ & 0.6989 \\
\hline Drowsiness, likes to lie down & $14(20.59)$ & $10(13.7)$ & 0.2767 \\
\hline Feels powerless and lazy & $30(44.12)$ & $28(38.36)$ & 0.4872 \\
\hline Looks powerless and lazy & $27(39.71)$ & $29(39.73)$ & 0.9981 \\
\hline Pale complexion & $6(8.82)$ & $5(6.85)$ & 0.6623 \\
\hline Reluctance to speak & $6(8.82)$ & $14(19.18)$ & 0.0782 \\
\hline Pale tongue & $14(20.59)$ & $12(16.44)$ & 0.5255 \\
\hline Teeth-marked tongue & $8(11.76)$ & $9(12.33)$ & 0.9181 \\
\hline Slow pulse & $7(10.29)$ & $13(17.81)$ & 0.2013 \\
\hline Weak pulse & $10(14.71)$ & $10(13.7)$ & 0.8640 \\
\hline Fine pulse & $9(13.24)$ & $1(1.37)$ & 0.0073 \\
\hline Reversed cold in the extremities & $7(10.29)$ & $7(9.59)$ & 0.8887 \\
\hline Sallow complexion & $20(29.41)$ & $23(31.51)$ & 0.7872 \\
\hline Dark inferior palpebral & $14(20.59)$ & $17(23.29)$ & 0.6989 \\
\hline Dizziness with nausea & $11(16.18)$ & $11(15.07)$ & 0.8562 \\
\hline White fur & $42(61.76)$ & $49(67.12)$ & 0.5063 \\
\hline Enlarged tongue & $12(17.65)$ & $10(13.7)$ & 0.5185 \\
\hline Slippery pulse & $26(38.24)$ & $40(54.79)$ & 0.0489 \\
\hline Heavy & $27(39.71)$ & $26(35.62)$ & 0.6164 \\
\hline
\end{tabular}

Data presented the number of subjects (\%) with symptoms/signs. $P$ value was calculated by chi-squared test or Fisher's test.

extracted ion chromatogram peak areas, organized by mass and retention time. Parameters for MarkerLynx analysis were set as follows: peak width at $5 \%$ height: $15 \mathrm{~s}$, intensity threshold: 20 , mass window: $0.1 \mathrm{amu}$, retention time window: $0.5 \mathrm{~min}$, noise elimination level: 6 , mass tolerance: $0.1 \mathrm{Da}$.
Normalization of MS spectra was performed by total normalization, and peaks not detected from over $2 \%$ of subjects were excluded in the following analysis. Metabolites were annotated using searches of the ChemSpider database (http://www.chemspider.com/) and Human Metabolome 
TABLE 3: List of metabolites exhibiting different levels in plasma between Dampness phlegm and non-Dampness phlegm.

\begin{tabular}{|c|c|c|c|c|}
\hline Retention time (min) & Mass $(m / z)$ & Non-DP & DP & $P$ \\
\hline \multicolumn{5}{|l|}{ ES $(+)$ mode } \\
\hline 20.04 & 1461.2680 & $6.61 \pm 1.43$ & $7.38 \pm 2.09$ & 0.009 \\
\hline 17.18 & 1039.724 & $153.13 \pm 44.87$ & $134.44 \pm 52.43$ & 0.010 \\
\hline 17.15 & 520.3663 & $304.62 \pm 48.29$ & $282.22 \pm 53.29$ & 0.015 \\
\hline 20.27 & 1494.291 & $18.50 \pm 4.16$ & $20.03 \pm 4.99$ & 0.022 \\
\hline 17.3 & 502.3353 & $15.82 \pm 5.11$ & $14.21 \pm 4.05$ & 0.024 \\
\hline 17.46 & 546.3991 & $41.14 \pm 17.33$ & $35.35 \pm 14.02$ & 0.034 \\
\hline 19.71 & 1376.208 & $20.36 \pm 6.07$ & $23.05 \pm 8.81$ & 0.037 \\
\hline 18.35 & 269.2497 & $4.13 \pm 1.26$ & $3.66 \pm 1.15$ & 0.042 \\
\hline 17.69 & 1043.763 & $113.36 \pm 46.83$ & $96.79 \pm 36.09$ & 0.044 \\
\hline 21.13 & 237.1001 & $26.71 \pm 11.22$ & $30.31 \pm 10.32$ & 0.046 \\
\hline 17.68 & 1565.1790 & $4.15 \pm 2.49$ & $3.33 \pm 1.53$ & 0.047 \\
\hline 29.01 & 195.0539 & $5.93 \pm 1.88$ & $6.57 \pm 1.97$ & 0.048 \\
\hline \multicolumn{5}{|l|}{ ES $(-)$ mode } \\
\hline 17.14 & 1019.5881 & $10.58 \pm 5.27$ & $8.77 \pm 5.09$ & 0.010 \\
\hline 0.6 & 444.6674 & $1.32 \pm 0.95$ & $1.55 \pm 0.82$ & 0.013 \\
\hline 0.53 & 740.8311 & $5.84 \pm 2.06$ & $6.63 \pm 2.25$ & 0.015 \\
\hline 17.5 & 590.3351 & $104.35 \pm 42.97$ & $88.28 \pm 38.06$ & 0.017 \\
\hline 29.23 & 599.3050 & $2.50 \pm 0.82$ & $2.20 \pm 0.69$ & 0.015 \\
\hline 29.23 & 288.1364 & $3.15 \pm 1.06$ & $3.47 \pm 1.01$ & 0.029 \\
\hline 0.53 & 462.8737 & $1.23 \pm 0.49$ & $1.44 \pm 0.59$ & 0.032 \\
\hline 17.53 & 658.3196 & $4.30 \pm 1.33$ & $3.89 \pm 0.98$ & 0.038 \\
\hline 19.87 & 961.5879 & $7.01 \pm 3.20$ & $8.21 \pm 3.71$ & 0.042 \\
\hline
\end{tabular}

Data are presented as mean normalized peak areas \pm SD. The $P$ value was calculated by Mann-Whitney $U$ test.

Database Version 2.5 (http://www.hmdb.ca/) or/and confirmed by external standards based on retention time and mass spectra.

2.6. Statistical Analysis. The statistical analysis of our data was performed with IBM SPSS Statistics 19 (IBM Co., New York, NC, USA). Normality of continuous variables in clinical data was decided by Kolmogorov-Smirnov test, and statistical difference was determined by $t$-test in parametric variable or Mann-Whitney $U$ test in nonparametric variable. Categorical variables were compared with a chi-squared test or Fisher's exact test. Results of MS analysis were represented as the mean $\pm \mathrm{SD}$, and the difference in mass intensity of plasma metabolites between the two groups was tested by independent $t$-test with Mann-Whitney $U$ test. The statistical significance was set at $P<0.05$.

\section{Results}

The general characteristics between non-DP and DP were shown in Table 1 . The mean age of the DP pattern patients was slightly higher than that of the non-DP pattern patients. BMI in body composition and total cholesterol and LDLcholesterol in serum parameters also showed higher levels in the DP pattern patients, which was similar to previous reports by Kim et al. and Lim et al. [3, 13]. The distribution of symptoms and signs according to "Korean Standard PIs for Stroke-III," which are parameters to decide the pattern of stroke [1], is shown in Table 2. Among pulse diagnoses, the ratio of subjects with slippery pulse in DP pattern was significantly higher $(54.8 \%)$ than that in non-DP pattern (38.2\%) $(P=0.0489)$. In contrast, the fraction of subjects exhibiting weak pulse was significantly lower in DP pattern $(P=0.0073)$.

To find metabolite levels in plasma that could differentiate non-DP and DP patterns, we performed UHPLCMS analysis (Figure 1). A list of metabolites present in significantly different levels in plasma of non-DP and DP patterns is presented in Table 3. Three abundant metabolites, detected as ions of $m / z 520.366,1039.724$, and 1043.763, in positive-ion mode and $m / z 590.335$ in negative-ion mode were significantly lower in the DP pattern relative to the non-DP pattern. However, most differentiating metabolites were present at much lower levels or were removed by the abundance threshold of 20 ion counts.

To identify each differentiating metabolite, public databases were searched using ion masses determined using both positive-ion and negative-ion modes. Phosphatidylcholine (PC) and lysophosphatidylcholine (LPC) lipids were identified from positive-ion mode data (Table 4). For example, the mean normalized peak areas of LPC $(18: 2 ; 9 Z$, $12 Z)$ and LPC $(20: 3)$, detected as $m / z 520.366$ and 546.399, were 282 and 35 in DP pattern and were significantly lower than 304 and 41 in non-DP pattern $(P=0.010$ and $P=0.034$, resp.). Additional LPCs with unsaturated fatty 
TABLE 4: Identification and quantification of metabolites in human plasma using UHPLC-MS and electrospray ionization in positive-ion mode.

\begin{tabular}{|c|c|c|c|c|c|c|c|c|}
\hline $\begin{array}{l}\text { Retention } \\
\text { time } \\
(\mathrm{min}) \\
\end{array}$ & Common name & $\begin{array}{l}\text { Formula of } \\
{[\mathrm{M}+\mathrm{H}]^{+}}\end{array}$ & $\begin{array}{c}\text { Measured } \\
\text { mass } \\
(m / z)\end{array}$ & $\begin{array}{c}\text { Theoretical } \\
\text { mass } \\
(\mathrm{m} / \mathrm{z})\end{array}$ & $\begin{array}{l}\text { Mass error } \\
\text { (Da) }\end{array}$ & Non-DP & DP & $P$ \\
\hline 16.55 & LPC(14:0) & $\mathrm{C}_{22} \mathrm{H}_{47} \mathrm{NO}_{7} \mathrm{P}^{+}$ & 468.35 & 468.31 & +0.04 & $21.87 \pm 11.51^{\mathrm{a}}$ & $22.95 \pm 9.12$ & 0.145 \\
\hline 16.86 & LPC(16:1 (9Z)) & $\mathrm{C}_{24} \mathrm{H}_{49} \mathrm{NO}_{7} \mathrm{P}^{+}$ & 494.36 & 494.32 & +0.04 & $58.80 \pm 35.99$ & $56.53 \pm 27.33$ & 0.671 \\
\hline 17.08 & LPC $(22: 6)$ & $\mathrm{C}_{30} \mathrm{H}_{51} \mathrm{NO}_{7} \mathrm{P}^{+}$ & 568.38 & 568.34 & +0.04 & $75.58 \pm 30.32$ & $77.35 \pm 37.30$ & 0.961 \\
\hline 17.12 & LPC $(20: 4)$ & $\mathrm{C}_{28} \mathrm{H}_{51} \mathrm{NO}_{7} \mathrm{P}^{+}$ & 544.37 & 544.34 & +0.03 & $104.65 \pm 31.01$ & $94.62 \pm 33.85$ & 0.070 \\
\hline 17.15 & LPC(18:2(9Z, 12Z)) & $\mathrm{C}_{26} \mathrm{H}_{51} \mathrm{NO}_{7} \mathrm{P}^{+}$ & 520.36 & 520.34 & +0.02 & $304.62 \pm 48.29$ & $282.22 \pm 53.29$ & 0.010 \\
\hline 17.15 & unknown & & 1063.75 & & & $45.49 \pm 17.50$ & $40.35 \pm 17.42$ & 0.058 \\
\hline 17.46 & $\operatorname{LPC}(20: 3)$ & $\mathrm{C}_{28} \mathrm{H}_{53} \mathrm{NO}_{7} \mathrm{P}^{+}$ & 546.39 & 546.35 & +0.04 & $41.14 \pm 17.33$ & $35.35 \pm 14.02$ & 0.034 \\
\hline 17.51 & $\operatorname{LPC}(16: 0)$ & $\mathrm{C}_{24} \mathrm{H}_{51} \mathrm{NO}_{7} \mathrm{P}^{+}$ & 496.36 & 496.34 & +0.02 & $530.37 \pm 56.28$ & $533.84 \pm 64.59$ & 0.583 \\
\hline 17.69 & LPC(18:1) & $\mathrm{C}_{26} \mathrm{H}_{53} \mathrm{NO}_{7} \mathrm{P}^{+}$ & 522.38 & 522.36 & +0.02 & $261.34 \pm 38.55$ & $249.65 \pm 39.38$ & 0.077 \\
\hline 18.21 & LPC $(18: 0)$ & $\mathrm{C}_{26} \mathrm{H}_{55} \mathrm{NO}_{7} \mathrm{P}^{+}$ & 524.39 & 524.37 & +0.02 & $398.11 \pm 41.16$ & $402.10 \pm 52.47$ & 0.391 \\
\hline 19.10 & $\begin{array}{c}\text { Dioctyl phthalate } \\
\text { (plasticizer contaminant) }\end{array}$ & $\mathrm{C}_{24} \mathrm{H}_{39} \mathrm{O}_{4}^{+}$ & 391.31 & 391.28 & +0.03 & $31.27 \pm 5.95$ & $31.27 \pm 4.95$ & 0.817 \\
\hline 19.38 & $\operatorname{DG}(40: 4)$ & $\mathrm{C}_{43} \mathrm{H}_{77} \mathrm{O}_{5}^{+}$ & 673.58 & 673.58 & +0.007 & $32.77 \pm 15.44$ & $36.78 \pm 19.95$ & 0.306 \\
\hline 19.67 & $\mathrm{DG}(40: 3)$ & $\mathrm{C}_{43} \mathrm{H}_{79} \mathrm{O}_{5}^{+}$ & 675.58 & 675.59 & -0.01 & $228.01 \pm 38.68$ & $229.73 \pm 45.96$ & 0.812 \\
\hline 19.73 & $\mathrm{DG}(42: 4)$ & $\mathrm{C}_{45} \mathrm{H}_{81} \mathrm{O}_{5}^{+}$ & 701.59 & 701.60 & -0.01 & $289.45 \pm 45.60$ & $289.82 \pm 44.59$ & 0.961 \\
\hline 19.85 & $\mathrm{PC}(36: 5)$ & $\mathrm{C}_{44} \mathrm{H}_{79} \mathrm{NO}_{8} \mathrm{P}^{+}$ & 780.59 & 780.55 & +0.04 & $213.70 \pm 45.71$ & $224.66 \pm 45.41$ & 0.156 \\
\hline 19.90 & $\mathrm{PC}(34: 3)$ & $\mathrm{C}_{42} \mathrm{H}_{79} \mathrm{NO}_{8} \mathrm{P}^{+}$ & 756.61 & 756.55 & +0.06 & $57.74 \pm 29.96$ & $58.82 \pm 28.32$ & 0.840 \\
\hline 19.95 & $\mathrm{PC}(38: 6)$ & $\mathrm{C}_{46} \mathrm{H}_{81} \mathrm{NO}_{8} \mathrm{P}^{+}$ & 806.61 & 806.56 & +0.05 & $219.88 \pm 37.58$ & $228.46 \pm 45.82$ & 0.501 \\
\hline 19.99 & $\mathrm{DG}(42: 3)$ & $\mathrm{C}_{45} \mathrm{H}_{83} \mathrm{O}_{5}^{+}$ & 703.61 & 703.62 & -0.01 & $172.78 \pm 29.19$ & $179.24 \pm 41.33$ & 0.564 \\
\hline 20.00 & Unknown & & 1485.24 & & & $63.66 \pm 25.34$ & $66.75 \pm 23.35$ & 0.536 \\
\hline 20.01 & $\mathrm{PC}(36: 4)$ & $\mathrm{C}_{44} \mathrm{H}_{81} \mathrm{NO}_{8} \mathrm{P}^{+}$ & 782.62 & 782.57 & +0.05 & $131.29 \pm 18.25$ & $128.28 \pm 22.48$ & 0.095 \\
\hline 20.08 & $\mathrm{PC}(38: 5)$ & $\mathrm{C}_{46} \mathrm{H}_{83} \mathrm{NO}_{8} \mathrm{P}^{+}$ & 808.66 & 808.59 & +0.07 & $30.93 \pm 11.17$ & $32.02 \pm 13.12$ & 0.760 \\
\hline 20.09 & $\mathrm{PC}(34: 2)$ & $\mathrm{C}_{42} \mathrm{H}_{81} \mathrm{NO}_{8} \mathrm{P}^{+}$ & 758.60 & 758.57 & +0.03 & $195.39 \pm 23.93$ & $197.90 \pm 27.36$ & 0.565 \\
\hline 20.15 & $\mathrm{PC}(36: 3)$ & $\mathrm{C}_{44} \mathrm{H}_{83} \mathrm{NO}_{8} \mathrm{P}^{+}$ & 784.63 & 784.58 & +0.045 & $135.50 \pm 23.95$ & $133.31 \pm 23.26$ & 0.300 \\
\hline 20.30 & $\mathrm{PC}(34: 1)$ & $\mathrm{C}_{42} \mathrm{H}_{83} \mathrm{NO}_{8} \mathrm{P}^{+}$ & 760.63 & 760.58 & +0.05 & $149.14 \pm 26.43$ & $154.41 \pm 26.00$ & 0.235 \\
\hline 20.32 & $\mathrm{PC}(38: 4)$ & $\mathrm{C}_{46} \mathrm{H}_{85} \mathrm{NO}_{8} \mathrm{P}^{+}$ & 810.68 & 810.60 & +0.08 & $49.85 \pm 13.02$ & $49.02 \pm 13.32$ & 0.638 \\
\hline 20.37 & $\operatorname{PC}(36: 2)$ & $\mathrm{C}_{44} \mathrm{H}_{85} \mathrm{NO}_{8} \mathrm{P}^{+}$ & 786.64 & 786.60 & +0.04 & $168.87 \pm 19.57$ & $170.71 \pm 25.72$ & 0.635 \\
\hline 20.43 & $\mathrm{PC}(38: 3)$ & $\mathrm{C}_{46} \mathrm{H}_{87} \mathrm{NO}_{8} \mathrm{P}^{+}$ & 812.68 & 812.62 & +0.06 & $60.73 \pm 26.71$ & $55.31 \pm 22.62$ & 0.149 \\
\hline 20.78 & $\mathrm{SM}(\mathrm{d} 18: 1 / 24: 1(15 \mathrm{Z}))$ & $\mathrm{C}_{47} \mathrm{H}_{94} \mathrm{~N}_{2} \mathrm{O}_{6} \mathrm{P}^{+}$ & 813.73 & 813.68 & +0.05 & $132.91 \pm 52.04$ & $131.24 \pm 59.93$ & 0.704 \\
\hline 20.79 & $\operatorname{SM}(\mathrm{d} 18: 0 / 22: 1(13 \mathrm{Z}))$ & $\mathrm{C}_{45} \mathrm{H}_{92} \mathrm{~N}_{2} \mathrm{O}_{6} \mathrm{P}^{+}$ & 787.72 & 787.67 & +0.05 & $50.88 \pm 40.64$ & $44.42 \pm 39.20$ & 0.217 \\
\hline
\end{tabular}

${ }^{a}$ Data are presented as mean \pm SD. The $P$ value was calculated by Mann-Whitney $U$ test. LPC: lysophosphatidylcholine; PC: phosphatidylcholine; DG: diacylglycerol; SM: sphingomyelin.

acid, LPC (20:4) and LPC (18:1), also showed a tendency to be lower in DP than non-DP pattern, but without significance ( $P=0.070$ and $P=0.77$, resp.). Otherwise, levels of LPCs without double bond, LPC (14:0), LPC (16:0), and LPC $(18: 0)$, were not different between two groups.

In the negative-ion mode data, a metabolite detected as $\mathrm{m} / z 590.335$ was significantly lower in DP pattern $(P=$ 0.017 ) and was identified as the formate ion adduct of LPC $(20: 3)$, confirming the result from positive-ion mode. Free fatty acids (FFA) and lysophosphatidylethanolamine (LPE), which were mainly detected in negative-ion mode analyses, were not different in the two patterns (Table 5). Only $\mathrm{LPE}(18: 2)$ showed a tendency to be lower in the DP pattern $(P=0.065)$.

\section{Discussion}

PI presents a comprehensive system for the diagnosis of disease and is based on the patient's signs and symptoms. TKM doctors determine the cause, nature, and treatment of the illness depending on signs and symptoms in patients and use this information to provide individualized treatment to patients, who have the same disease [3]. This approach is similar to what has been described as personalized medicine, which has recently emphasized that patients should receive different treatments according to the patient's individual condition to increase the positive effect of treatment and reduce adverse effects $[25,26]$. But general concepts between $\mathrm{PI}$ and personalized medicine are different. PI focuses on the 
TABLE 5: Identification and quantification of metabolites in human plasma using UHPLC-MS and electrospray ionization in negative-ion mode.

\begin{tabular}{|c|c|c|c|c|c|c|c|c|}
\hline $\begin{array}{l}\text { Retention } \\
\text { time (min) }\end{array}$ & Common name & $\begin{array}{l}\text { Formula of } \\
{[\mathrm{M}-\mathrm{H}]^{-}}\end{array}$ & $\begin{array}{c}\text { Measured } \\
\text { mass } \\
(m / z)\end{array}$ & $\begin{array}{c}\text { Theoretical } \\
\text { mass } \\
(\mathrm{m} / \mathrm{z})\end{array}$ & $\begin{array}{l}\text { Mass error } \\
\quad(\mathrm{Da})\end{array}$ & Non-DP & $\mathrm{DP}$ & $P$ \\
\hline 0.64 & unknown & & 291.08 & & & $122.96 \pm 70.25^{\mathrm{a}}$ & $136.14 \pm 107.74$ & 0.869 \\
\hline 17.10 & $\operatorname{LPE}(22: 6)$ & $\mathrm{C}_{27} \mathrm{H}_{43} \mathrm{NO}_{7} \mathrm{P}^{-}$ & 524.27 & 524.28 & $<0.01$ & $65.99 \pm 30.35$ & $61.52 \pm 25.40$ & 0.419 \\
\hline 17.17 & $\operatorname{LPE}(20: 4)$ & $\mathrm{C}_{25} \mathrm{H}_{43} \mathrm{NO}_{7} \mathrm{P}^{-}$ & 500.27 & 500.27 & $<0.01$ & $45.65 \pm 20.26$ & $40.26 \pm 17.24$ & 0.065 \\
\hline 17.23 & $\operatorname{LPE}(18: 2)$ & $\mathrm{C}_{23} \mathrm{H}_{43} \mathrm{NO}_{7} \mathrm{P}^{-}$ & 476.27 & 476.28 & $<0.01$ & $70.53 \pm 34.49$ & $65.03 \pm 35.97$ & 0.289 \\
\hline 17.69 & $\operatorname{LPE}(16: 0)$ & $\mathrm{C}_{21} \mathrm{H}_{43} \mathrm{NO}_{7} \mathrm{P}^{-}$ & 452.27 & 452.28 & $<0.01$ & $48.60 \pm 17.28$ & $46.68 \pm 18.35$ & 0.258 \\
\hline 17.88 & LPE(18:1) & $\mathrm{C}_{23} \mathrm{H}_{45} \mathrm{NO}_{7} \mathrm{P}^{-}$ & 478.29 & 478.29 & $<0.01$ & $27.78 \pm 18.78$ & $24.01 \pm 12.01$ & 0.409 \\
\hline 18.32 & Eicosapentaenoic acid (C20:5) & $\mathrm{C}_{20} \mathrm{H}_{29} \mathrm{O}_{2}^{-}$ & 301.21 & 301.22 & $<0.01$ & $47.59 \pm 31.47$ & $48.31 \pm 27.00$ & 0.520 \\
\hline 18.37 & Linolenic acid (C18:3) & $\mathrm{C}_{18} \mathrm{H}_{29} \mathrm{O}_{2}^{-}$ & 277.21 & 277.22 & $<0.01$ & $58.12 \pm 50.50$ & $64.84 \pm 64.66$ & 0.495 \\
\hline 18.64 & Docosahexaenoic acid (C22:6) & $\mathrm{C}_{22} \mathrm{H}_{31} \mathrm{O}_{2}^{-}$ & 327.23 & 327.23 & $<0.01$ & $268.96 \pm 121.50$ & $294.55 \pm 116.64$ & 0.093 \\
\hline 18.68 & Free fatty acid (C16:1) & $\mathrm{C}_{16} \mathrm{H}_{29} \mathrm{O}_{2}^{-}$ & 253.21 & 253.22 & $<0.01$ & $83.04 \pm 78.33$ & $74.83 \pm 63.94$ & 0.901 \\
\hline 18.77 & Arachidonic acid (C20:4) & $\mathrm{C}_{20} \mathrm{H}_{31} \mathrm{O}_{2}^{-}$ & 303.23 & 303.23 & $<0.01$ & $161.10 \pm 72.52$ & $149.19 \pm 77.86$ & 0.306 \\
\hline 18.86 & Linoleic acid (C18:2) & $\mathrm{C}_{18} \mathrm{H}_{31} \mathrm{O}_{2}^{-}$ & 279.23 & 279.23 & $<0.01$ & $276.78 \pm 191.57$ & $279.87 \pm 161.58$ & 0.635 \\
\hline 18.93 & $\begin{array}{c}\text { Docosapentaenoic acid } \\
\text { (C22:5) }\end{array}$ & $\mathrm{C}_{22} \mathrm{H}_{33} \mathrm{O}_{2}^{-}$ & 329.25 & 329.25 & $<0.01$ & $32.17 \pm 23.16$ & $31.04 \pm 18.84$ & 0.749 \\
\hline 19.04 & Free fatty acid (C20:3) & $\mathrm{C}_{20} \mathrm{H}_{33} \mathrm{O}_{2}^{-}$ & 305.25 & 305.25 & $<0.01$ & $29.70 \pm 19.08$ & $29.47 \pm 22.32$ & 0.612 \\
\hline 19.18 & $\operatorname{DG}(30: 1)$ & $\mathrm{C}_{33} \mathrm{H}_{61} \mathrm{O}_{5}^{-}$ & 537.41 & 537.45 & -0.04 & $17.87 \pm 9.91$ & $19.65 \pm 9.45$ & 0.176 \\
\hline 19.27 & Palmitic acid (C16:0) & $\mathrm{C}_{16} \mathrm{H}_{31} \mathrm{O}_{2}^{-}$ & 255.23 & 255.23 & $<0.01$ & $218.29 \pm 140.27$ & $209.29 \pm 147.29$ & 0.626 \\
\hline 19.32 & $\mathrm{DG}(30: 0)$ & $\mathrm{C}_{33} \mathrm{H}_{63} \mathrm{O}_{5}^{-}$ & 539.42 & 539.46 & -0.04 & $11.81 \pm 7.63$ & $15.08 \pm 16.86$ & 0.440 \\
\hline 19.36 & Oleic acid (C18:1) & $\mathrm{C}_{18} \mathrm{H}_{33} \mathrm{O}_{2}^{-}$ & 281.25 & 281.25 & $<0.01$ & $402.57 \pm 311.69$ & $378.81 \pm 270.00$ & 0.875 \\
\hline 19.55 & unknown & & 393.27 & & & $63.50 \pm 20.78$ & $65.85 \pm 22.13$ & 0.641 \\
\hline 19.57 & $\operatorname{MG}(22: 5)$ & $\mathrm{C}_{25} \mathrm{H}_{39} \mathrm{O}_{4}^{-}$ & 403.30 & 403.28 & +0.02 & $69.14 \pm 27.41$ & $67.50 \pm 31.95$ & 0.405 \\
\hline 19.62 & Free fatty acid (C17:0) & $\mathrm{C}_{17} \mathrm{H}_{33} \mathrm{O}_{2}^{-}$ & 269.24 & 269.25 & -0.01 & $20.24 \pm 13.09$ & $21.04 \pm 14.72$ & 0.862 \\
\hline 19.86 & unknown & & 364.49 & & & $54.03 \pm 18.02$ & $49.21 \pm 15.44$ & 0.054 \\
\hline 19.89 & Stearic acid $(\mathrm{C} 18: 0)$ & $\mathrm{C}_{18} \mathrm{H}_{35} \mathrm{O}_{2}^{-}$ & 283.261 & 283.264 & -0.003 & $243.29 \pm 118.20$ & $220.01 \pm 101.87$ & 0.212 \\
\hline
\end{tabular}

${ }^{a}$ Data are presented as mean \pm SD. The $P$ value was calculated by Mann-Whitney $U$-test. LPE: lysophosphatidylethanolamine; DG: diacylglycerol, MG: monoacylglycerol; PE: phosphatidylethanolamine.

signs and symptoms in patients to define a phenotype that guides treatment selection. Much of the emphasis of modern personalized medicine is based on the genetic background, or genotype, of each patient $[3,27]$. Therefore, studies to establish relationships between PI and personalized medicine have been performed $[10,11,13]$.

In TKM, stroke is classified into four standard subtypes according to PI: QD, DP, YD, and FH based on position, internal or external etiology, or observations of affected organ. DP, which is classified by etiology, is a combination of phlegm and internal dampness causing disease. Major symptoms of the DP pattern include anorexia, dyspepsia, dizziness, headache, and heaviness [28]. However, not all patients with DP pattern have all of these symptoms, and subjects with other patterns often exhibit some of the same symptoms. For example, only $15 \%$ of subjects had dizziness with nausea, considered a major indicator in the DP pattern, and this ratio was not different in subjects with non-DP pattern. Slippery pulse, which is an important factor for the diagnosis of DP pattern and significantly high ratio at DP pattern, occurred in the
non-DP pattern (Table 2). These complexities of sign and symptoms complicate PI diagnosis and may cause diagnostic error between TKM doctors. Previous studies reported that concordance rates between two expert TKM doctors about PI diagnosis for stroke, pulse, or tongue diagnosis were 70$90 \%$, which means that the diagnostic error may always be implied [29-31]. For these reasons, reliable biomarkers for more accurate diagnosis of PI are needed.

Metabolomics provides powerful tools to measure and identify metabolic biomarkers from biofluids, and earlier studies to explain PI using the change in metabolite levels were performed in China [21, 22]. In this study, we elucidated UHPLC-MS analysis from plasma of the subjects with CI to find metabolites related with DP pattern of stroke (Figure 1). From the total of 612 peaks corresponding to specific mass-retention time pairs, 21 metabolite peaks showed significant differences in the levels between DP and non-DP patterns (Table 3). In addition, searches of open metabolome databases allowed annotation of 57 major metabolites detected using this protocol (Tables 4 and 5). 
The primary conclusions are that the levels of LPC $(18: 2,9 \mathrm{Z}$, $12 Z)$ and LPC $(20: 3)$ were significantly lower in DP pattern than non-DP pattern $(P=0.010$ and 0.034 , resp.), and LPC $(20: 4)$ and LPC $(18: 1)$ showed a tendency to decrease in DP pattern $(P=0.070$ and $P=0.077)$.

Lysophosphatidylcholines (LPCs), called lysolecithins, are a class of phospholipids derived from phosphatidylcholines and produced by two pathways. First is the result from partial hydrolysis of phosphatidylcholine, so that one of the fatty acids is removed by the action of phospholipase A2 [32]. A second pathway for LPC formation occurs by the transfer of one fatty acid of phosphatidylcholine to cholesterol by lecithin-cholesterol acyltransferase (LCAT), which is an enzyme that converts free cholesterol into cholesteryl ester. Cholesteryl ester is a more hydrophobic form of cholesterol that is sequestered into the core of a lipoprotein particle [33] and into liver.

Until now, relationships between plasma levels of LPCs and DP pattern were not known, but earlier reports suggested the possibility of such correlations. It was known that DP pattern was related to obesity and hyperlipidemia $[3,8,9]$, and some metabolomic analyses showed that plasma LPC levels were also associated with obesity [34-37]. Barber et al. and Kim et al. reported that plasma levels of LPCs with one or two double bonds such as lyso (16:1), LPC (18:1), LPC $(18: 2)$, and LPC $(20: 1)$ were significantly reduced in highfat diet-induced obese mice [35, 36], In another study among overweight/obese subjects performed by Kim et al., LPC $(18: 1)$, LPC $(18: 2)$, and LPC $(20: 2)$ were decreased in the plasma of overweight/obese subjects [37]. Of particular note in these studies was the saturated LPC $(18: 0)$, that increased in obese subjects and obese mice model [36,37], and these results parallel the findings in the current investigation (Table 4).

This study showed, for the first time, that some LPCs in plasma were associated with DP pattern in CI patient population. However, this study included several limitations. First, this is a simple cross-sectional study, and the subjects enrolled in this study were very acute patients. Second, this study does not have a sufficient sample size to generalize the relationship between plasma metabolites and DP pattern. Third, the PI was limited to stroke diagnoses. The lack of evidence on the accuracy of PI forced us to perform an observational study, not allowing a randomized controlled trial. Despite of these limitations, this study provided metabolic information about DP patterns, and further studies should be performed in subjects of another large population to generalize the conclusions of this study.

\section{Authors' Contribution}

M. H. Cha and A. D. Jones contributed equally to this work.

\section{Acknowledgments}

This research was supported by a Grant from the Korea Institute of Oriental Medicine (K12130) and funds from Michigan AgBioResearch.

\section{References}

[1] J. A. Lee, J. S. Lee, B. K. Kang et al., "Report on the Korean standard pattern identifications for stroke-III," Korean Journal of Oriental Internal Medicine, vol. 32, pp. 232-242, 2011.

[2] H. Y. Go, Y. K. Kim, B. K. Kang et al., "Report on the Korean standard differentiation of the symptoms and signs for the stroke-2," Korean Journal of Oriental Physiology \& Pathology, vol. 20, pp. 1789-1791, 2006.

[3] H. J. Kim, H. S. Bae, S. U. Park, S. K. Moon, J. M. Park, and W. S. Jung, "Clinical approach to the standardization of oriental medical diagnostic pattern identification in stroke patients," Evidence-Based Complementary and Alternative Medicine, vol. 2011, Article ID 768492, 7 pages, 2011.

[4] W. W. Jung and W. C. Lee, "The literature on "Dam-eum" resulted on stroke," Dongguk Journal of the Institute of Oriental Medicine, vol. 8, pp. 133-144, 1998.

[5] S. Y. Kim, J. S. Lee, B. K. Kang et al., "Study on the relationship among Bi-Su type, obesity index and pattern identification in Korean stroke patients," Korean Journal of Oriental Internal Medicine, vol. 30, pp. 550-557, 2009.

[6] J. S. Kang, D. H. Kim, H. S. Shin et al., “The study on relationship of dampness-phlegm tongue diagnosis to hyperlippidemia in stroke patients," The Journal of the Korea Institute of Oriental Medical Diagnosis, vol. 13, pp. 24-33, 2009.

[7] M. H. Cha, S. Y. Kim, J. H. Lim et al., "Study on the obesity and blood parameters differences between fire/heat and Qi-deficiency pattern identification/syndrome differentiation among acute stroke patient," Korean Journal of Oriental Institute Medicine, vol. 30, no. 4, pp. 772-779, 2009.

[8] J. H. Lim, M. M. Ko, J. S. Lee et al., "Genetic association of SNPs located at PON1 gene with dampness and phlegm pattern identification among Korea stroke patients," Korean Journal of Oriental Internal Medicine, vol. 31, pp. 752-762, 2010.

[9] M. M. Ko, B. K. Kang, J. H. Lim, M. S. Lee, and M. H. Cha, "Genetic association of NPY gene polymorphisms with dampness-phlegm pattern in Korean stroke patients," Evidence-Based Complementary and Alternative Medicine, vol. 2012, Article ID 109796, 7 pages, 2012.

[10] M. M. Ko, T. Y. Park, J. H. Lim, M. H. Cha, and M. S. Lee, "WNT10B polymorphism in Korean stroke patients with Yin deficiency pattern," Evidence-Based Complementary and Alternative Medicine, vol. 2012, Article ID 798131, 6 pages, 2012.

[11] Y. Wu, Y. Cun, J. Dong et al., "Polymorphisms in PPARD, PPARG and APM1 associated with four types of traditional Chinese medicine constitutions," Journal of Genetics and Genomics, vol. 37, no. 6, pp. 371-379, 2010.

[12] J. H. Lim, M. M. Ko, J. S. Lee et al., "Different level of plasma free hemoglobin between Qi-deficiency and fire heat among Korean stoke subjects," Korean Journal of Oriental Physiology \& Pathology, vol. 25, no. 4, pp. 697-701, 2011.

[13] J. H. Lim, M. M. Ko, H. Lee et al., "Differential association of uncoupling protein 2 polymorphisms with pattern identification among Korean stroke patients: a diagnostic system in traditional Korean medicine," Evidence-Based Complementary and Alternative Medicine, vol. 2012, Article ID 532078, 9 pages, 2012.

[14] J. K. Nicholson and J. C. Lindon, "Systems biology: metabonomics," Nature, vol. 455, no. 7216, pp. 1054-1056, 2008.

[15] J. L. Spratlin, N. J. Serkova, and S. G. Eckhardt, "Clinical applications of metabolomics in oncology: a review," Clinical Cancer Research, vol. 15, no. 2, pp. 431-440, 2009. 
[16] W. M. Claudino, A. Quattrone, L. Biganzoli, M. Pestrin, I. Bertini, and A. Di Leo, "Metabolomics: available results, current research projects in breast cancer, and future applications," Journal of Clinical Oncology, vol. 25, no. 19, pp. 2840-2846, 2007.

[17] Y. Qiu, G. Cai, M. Su et al., "Serum metabolite profiling of human colorectal cancer using GC-TOFMS and UPLCQTOFMS," Journal of Proteome Research, vol. 8, no. 10, pp. 4844-4850, 2009.

[18] S. H. Shah, J. L. Sun, R. D. Stevens et al., "Baseline metabolomic profiles predict cardiovascular events in patients at risk for coronary artery disease," American Heart Journal, vol. 163, no. 5, pp. 844-850, 2012.

[19] M. Ciborowski, J. Teul, J. L. Martin-Ventura, J. Egido, and C. Barbas, "Metabolomics with LC-QTOF-MS permits the prediction of disease stage in aortic abdominal aneurysm based on plasma metabolic fingerprint," PLoS One, vol. 7, no. 2, Article ID e31982, 2012.

[20] J. Y. Jung, H. S. Lee, D. G. Kang et al., "1 H-NMR-based metabolomics study of cerebral infarction," Stroke, vol. 42, no. 5, pp. 1282-1288, 2011.

[21] Y. Gu, C. Lu, Q. Zha et al., "Plasma metabonomics study of rheumatoid arthritis and its Chinese medicine subtypes by using liquid chromatography and gas chromatography coupled with mass spectrometry," Molecular Biosystems, vol. 8, no. 5, pp. 1535-1543, 2012.

[22] H. Wei, W. Pasman, C. Rubingh et al., "Urine metabolomics combined with the personalized diagnosis guided by Chinese medicine reveals subtypes of pre-diabetes," Molecular Biosystems, vol. 8, no. 5, pp. 1482-1492, 2012.

[23] T. Y. Park, J. A. Lee, M. H. Cha et al., "The fundamental study for the standardization and objectification of pattern identification in traditional Korean medicine for stroke (SOPI-Stroke): an overview of phase I," European Journal of Integrative Medicine, vol. 4, pp. e125-e131, 2012.

[24] W. B. Dunn, D. Broadhurst, P. Begley et al., "Procedures for large-scale metabolic profiling of serum and plasma using gas chromatography and liquid chromatography coupled to mass spectrometry," Nature Protocols, vol. 6, no. 7, pp. 1060-1083, 2011.

[25] I. Kirstein-Grossman, J. S. Beckmann, D. Lancet, and A. Miller, "Pharmacogenetic development of personalized medicine: multiple sclerosis treatment as a model," Drug News and Perspectives, vol. 15, no. 9, pp. 558-567, 2002.

[26] A. H. Trainer, B. Meiser, K. Watts, G. Mitchell, K. Tucker, and M. Friedlander, "Moving toward personalized medicine: treatment-focused genetic testing of women newly diagnosed with ovarian cancer," International Journal of Gynecological Cancer, vol. 20, no. 5, pp. 704-716, 2010.

[27] K. E. Morrison, "Whole-genome sequencing informs treatment: personalized medicine takes another step forward," Clinical Chemistry, vol. 57, no. 12, pp. 1638-1640, 2011.

[28] WHO, WHO International Standard Terminologies on Traditional Medicine in the Western Pacific Region, World Health Organization, 2007.

[29] B. K. Kang, K. K. Kang, S. W. Park et al., "The discrimination model for the pattern identification diagnosis of the stroke," Korean Journal of Oriental Internal Medicine, vol. 13, pp. 59-63, 2007.

[30] M. M. Ko, T. Y. Park, J. A. Lee, T. Y. Choi, B. K. Kang, and M. S. Lee, "Interobserver reliability of pulse diagnosis using traditional Korean medicine for stroke patients," The Journal of
Anternative and Complementary Medicine, vol. 19, no. 1, pp. 2934, 2013.

[31] B. K. Kang, T. Y. Park, J. A. Lee et al., "Reliability and validity of the Korean standard pattern identification for stroke (K-SPIStroke) questionnaire," BMC Complementary and Alternative Medicine, vol. 12, article 55, 2012.

[32] T. Matsumoto, T. Kobayashi, and K. Kamata, "Role of lysophosphatidylcholine (LPC) in atherosclerosis," Current Medicinal Chemistry, vol. 14, no. 30, pp. 3209-3220, 2007.

[33] J. A. Kuivenhoven, H. Pritchard, J. Hill, J. Frohlich, G. Assmann, and J. Kastelein, "The molecular pathology of lecithin: cholesterol acyltransferase (LCAT) deficiency syndromes," Journal of Lipid Research, vol. 38, no. 2, pp. 191-205, 1997.

[34] K. H. Pietiläinen, M. Sysi-Aho, A. Rissanen et al., "Acquired obesity is associated with changes in the serum lipidomic profile independent of genetic effects-a monozygotic twin study," PLoS One, vol. 2, no. 2, article e218, 2007.

[35] M. N. Barber, S. Risis, C. Yang et al., "Plasma lysophosphatidylcholine levels are reduced in obesity and type 2 diabetes," PLoS One, vol. 7, no. 7, Article ID e41456, 2012.

[36] H. J. Kim, J. H. Kim, S. Noh et al., "Metabolomic analysis of livers and serum from high-fat diet induced obese mice," Journal of Proteome Research, vol. 10, no. 2, pp. 722-731, 2011.

[37] J. Y. Kim, J. Y. Park, O. Y. Kim et al., "Metabolic profiling of plasma in overweight/obese and lean men using ultra performance liquid chromatography and Q-TOF Mass spectrometry (UPLC-Q-TOF MS)," Journal of Proteome Research, vol. 9, no. 9, pp. 4368-4375, 2010. 


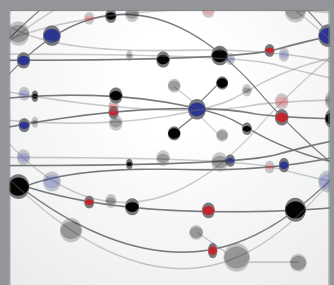

The Scientific World Journal
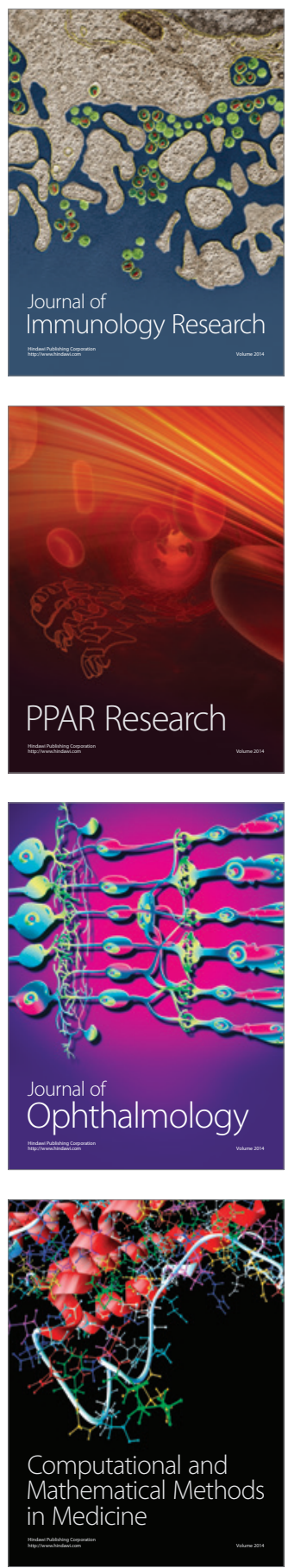

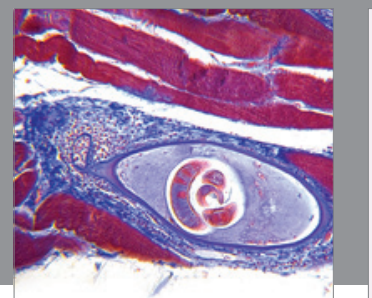

Gastroenterology

Research and Practice
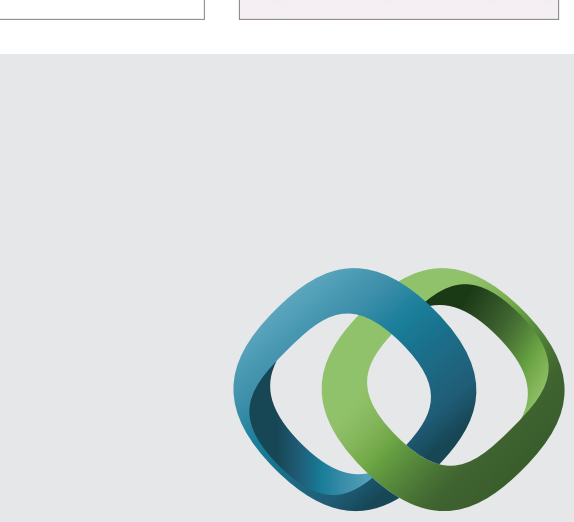

\section{Hindawi}

Submit your manuscripts at

http://www.hindawi.com
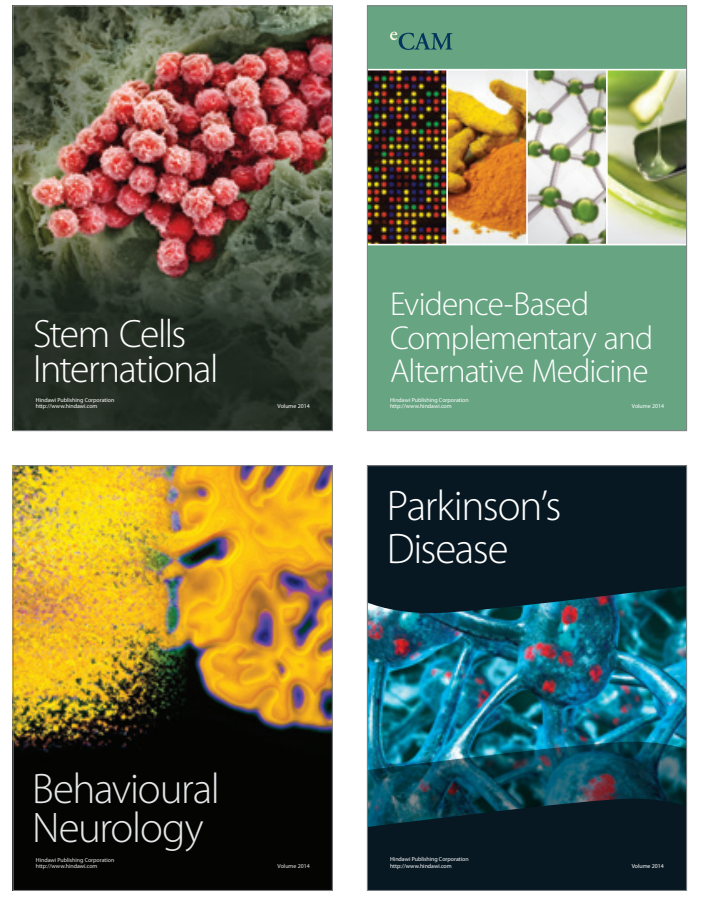
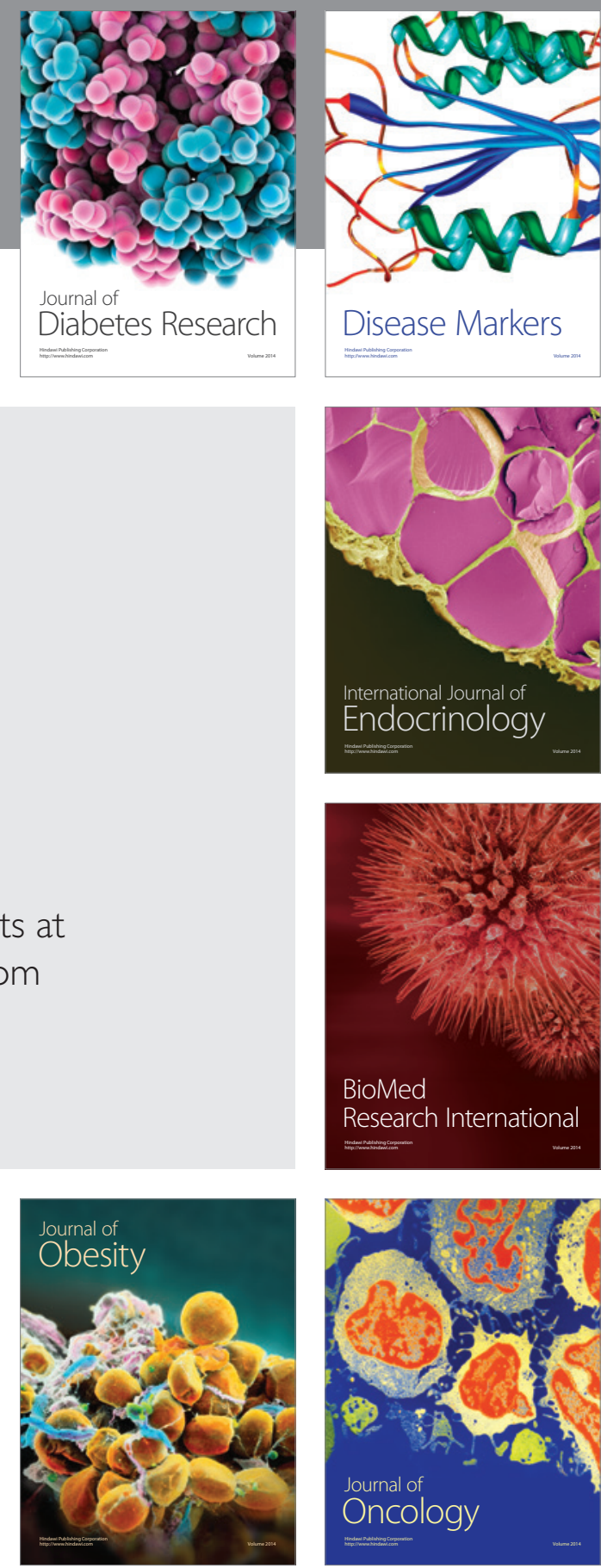

Disease Markers
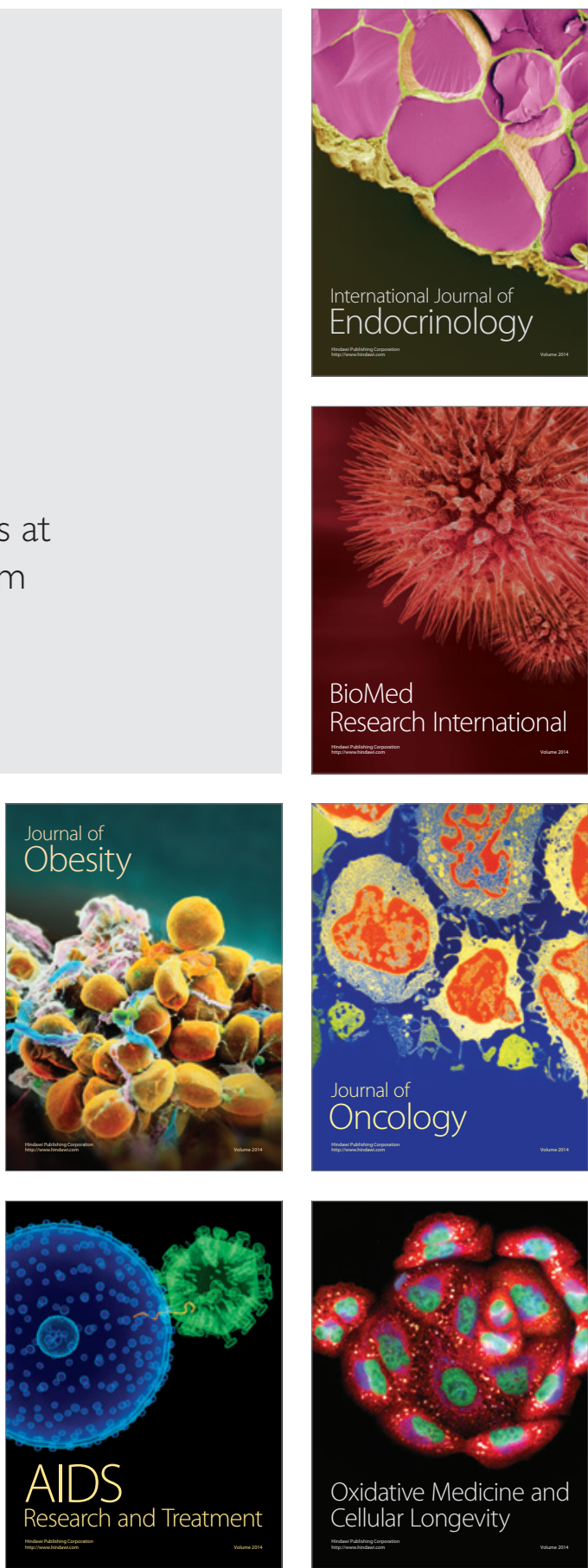